\title{
Ultrasound influence on exciton emission of GaP light diodes
}

\author{
O.M. Gontaruk ${ }^{1}$, V.I. Khivrych ${ }^{1}$, M.B. Pinkovska1 ${ }^{1}$, V.P. Tartachnyk ${ }^{1}$, Ya.M. Olikh ${ }^{2}$, \\ R.M. Vernydub ${ }^{3}$, V.Ya. Opilat ${ }^{3}$ \\ ${ }^{1}$ Institute for Nuclear Research, 47 prospect Nauky, Kyiv-28, Ukraine \\ ${ }^{2}$ Institute of Semiconductor Physics, NAS of Ukraine, 45 prospect Nauky, Kyiv-28, Ukraine \\ ${ }^{3}$ The Dragomanov National Pedagogical University, 9 Pirogova str., Kyiv-23, Ukraine
}

\begin{abstract}
Electroluminescence of GaP light diodes, treated by ultrasound at room and low temperatures has been studied. It has been found that short ultrasound caused improvement of red diode emission characteristics while electroluminescence degradation occurred in structures treated by prolong treatment at room temperature. Spectral curves measured under acoustic dynamic mode possess some specific features concerning fine structure. If reverse biased diodes possess microplasma initially, ultrasound action decreases its brightness. Possible mechanisms of the ultrasonic interaction with crystal structure defects have been discussed.
\end{abstract}

Keywords: electroluminescence, ultrasound, degradation, relaxation, dislocation, exciton, microplasma.

Paper received 18.03.03; accepted for publication 16.06.03.

\section{Introduction}

It is known that ultrasound treatment of semiconductors and semiconductor devices may promote improvement of their parameters. Processes which lead to the increase of luminescence intensity, light diodes quantum yield and current carrier drift mobility while noise decreases, sample hardness and semiconductor device reliability [1] are mainly based on the partial structure refinement and inner stress decrease [2].

The mechanism of ultrasound interaction with structure defects in $\mathrm{GaP}$, one of the most important devices of optoelectronics, is not clear so far and different models of the explanation of the phenomena observed are involved.

The goal of the paper presented was to obtain some information about the luminescence degradation mechanism in GaP and to study the possibility to improve radiative emittance of ultrasound treated red light diodes.

\section{Methods and results}

GaP samples investigated were a sort of $p$ - $n$ structures without shell, grown by the double liquid epitaxy method: $n$-region was doped by Te to the concentration of $8 \cdot 10^{17} \mathrm{~cm}^{-3}$; $p$ - region - by $\mathrm{Zn}$ and $\mathrm{O}$ simultaneously $\left(p \sim 3 \cdot 10^{17} \mathrm{~cm}^{-3}\right)$, Electroluminescence intensity of structures at room and low temperatures has been measured. Samples have been treated by ultrasound with frequencies $3.1-7.0 \mathrm{MHz}$, the acoustic wave power $W \leq 0.5 \mathrm{Wcm}^{-2}$.

Electroluminescence spectrum at room temperature is a broad band $(h v=1.81 \mathrm{eV})$ caused by recombination of excitons bounded on $\mathrm{Zn}-\mathrm{O}$ pairs. At the low temperature $(77 \mathrm{~K})$ an additional luminescence band $h v=2.1 \mathrm{eV}$ appears.

It has been found that ultrasound treatment of light diodes at room temperature during short period of time $(t<10 \mathrm{~min}$.) causes the slight increase (to $5 \%$ ) of electroluminescence in the band maximum $h v=1.8 \mathrm{eV}$. Subsequent ultrasound treatment leads to the monotonous decrease of the emission intensity, which gradually restores to the initial value after ultrasound stoppage with time of some decades of minutes (Fig. 1). Under ultrasound treatment with the time more than $t=4.5 \mathrm{~h}$ a curve with many separated lines is observed (Fig. 2).

If a reverse biased sample initially possesses the microplasma, ultrasound causes the decrease of its brightness. 
O.M. Gontaruk et al.: Ultrasound influence on exciton emission of GaP light ...

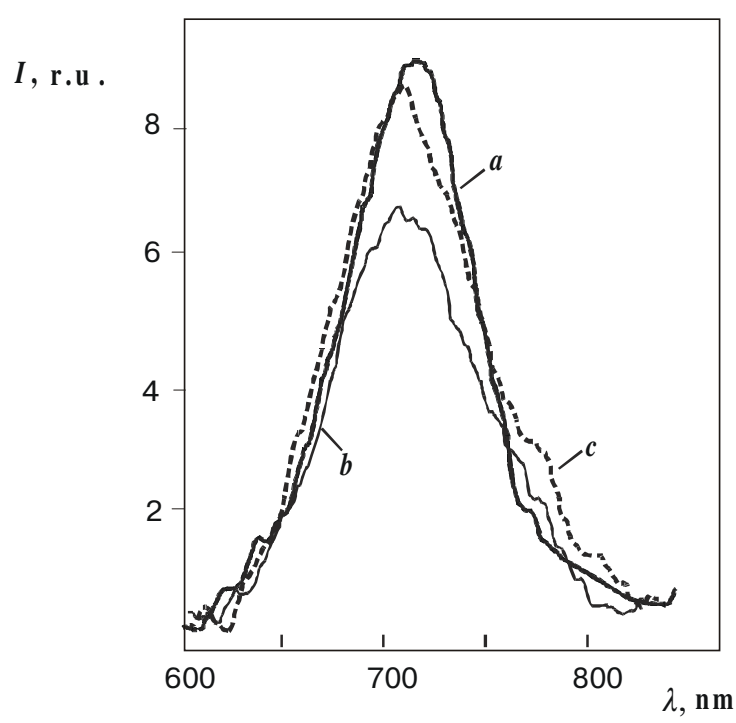

Fig. 1. Luminescence spectra: $a$ - initial sample; $b$-ultrasound treated; $c$-after keeping at the room temperature for $90 \mathrm{~min}$

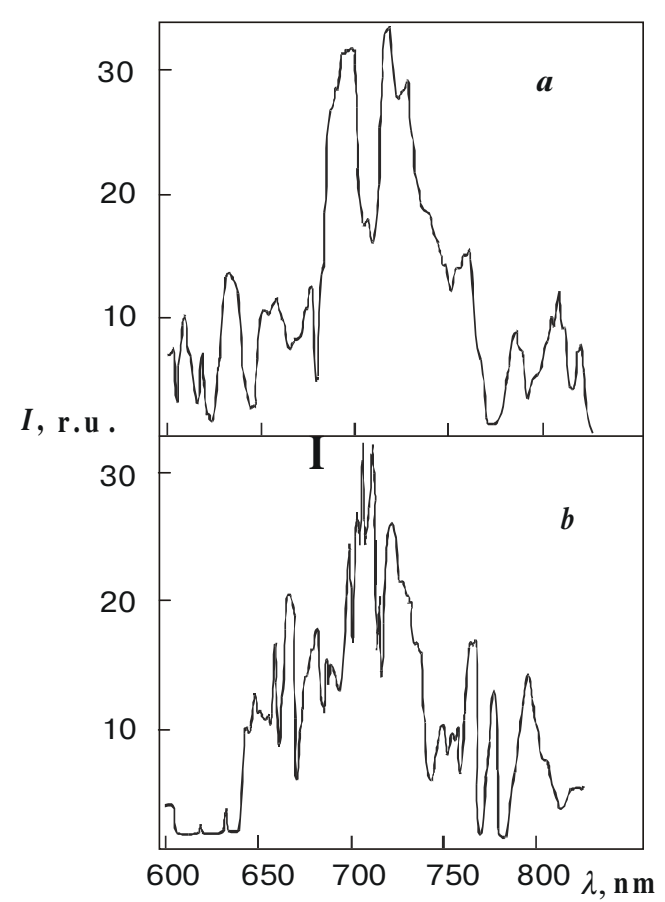

Fig. 2. Luminescence spectra after ultrasound treatment for: $a-$ 60 min., $b-4.5 \mathrm{~h}$.

Electroluminescence measured at dynamic mode had shown essential changes of the structure: intensive wandering bands ( which were presented in the spectrum of degraded samples treated by ultrasound for a long time) disappeared and the curve recalled initial spectra (Fig. 3). Such restoring ultrasound action is especially effective at $77 \mathrm{~K}$. The ultrasound stoppage causes the returning (during some minutes) of diode characteristics to the previous state, typical for degraded samples. The spectral characteristics improvement in the acoustodynamic mode is thus the temporary effect, which occurs only under ultrasound action.

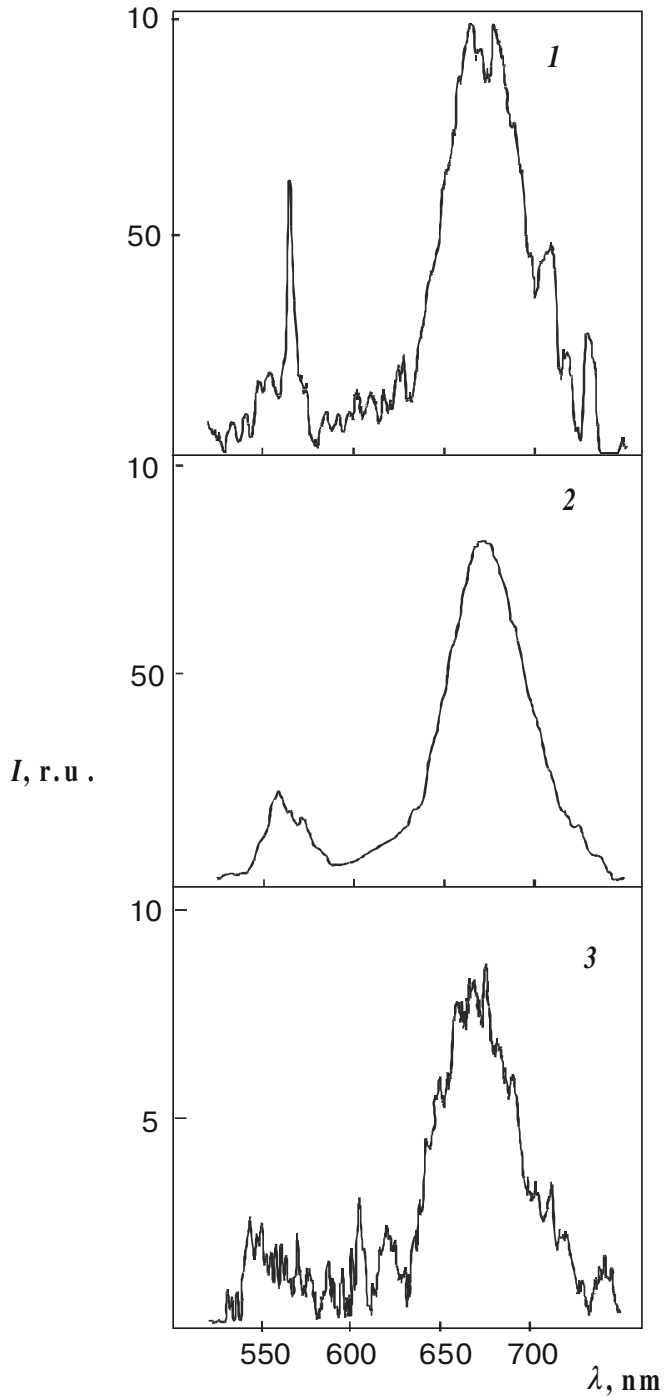

Fig. 3. Electroluminescence spectrum: $I$ - degraded light emitting diode; 2 - ultrasound treated sample at $77 \mathrm{~K}$ (dynamic mode); 3 - the same diode after ultrasound stoppage.

\section{Discussion}

Let us consider possible mechanisms of ultrasound influence on the crystal structure.

I. Mechanism of the ultrasound stimulated capture of point defects by dislocation. The acoustostimulated capture of mobile point defects by linear defects (dislocations, grain boundaries, precipitates) has been experimentally observed in different semiconductors [2]. This phenomenon is based on the mechanism of ultrasound influence on point defect diffusion coefficients. Thus, it has been shown by Bakaj and Lazarev [3] that the inner tension field of the ultrasound wave can essentially change atomic diffusion coefficients in crystals. As one can see from results of Dobrovolski and others [4], the piesofield amplitude reaches the value of some decades of $\mathrm{kV} / \mathrm{cm}$ and thus an atom movement can be essentially accelerated. The increase and as well the decrease of dif- 
O.M. Gontaruk et al.: Ultrasound influence on exciton emission of GaP light ...

fusion coefficients has been observed for different materials in practice.

Possibilities of the crystal "temporary" clearing out of point defects due to the atom capture by dislocations, oscillated with frequency w in acoustic wave field, can be estimated in the next procedure.

Wandering throughout the lattice, a separate atom jumps with the time $t_{\boldsymbol{a}}[5]$ :

$\tau_{a} \approx 10^{-13} e^{E_{m} / k T} e^{S_{m} / k}$,

where $S_{m} / k \approx 1$ (for metal vacancy). $S_{m}-$ is the entropy, $E_{m}$-defect migration energy. This value is as a rule a bit less than activation energy $\mathrm{E}_{\mathrm{a}}$.

It has been shown [1] that coefficient of impurity diffusion along dislocation in germanium and silicon is $3 \div 4$ orders higher than a volume diffusion coefficient. Activation energy of impurity is equal 0.8 value of volume energy $E_{a}$. In the case of the dislocation incorporation the simultaneous influence of both factors (the increase of impurity atom diffusion coefficient and the decrease of activation energy) may cause the increase of the volume diffusion coefficient by several orders of value.

So the dislocation influence on the diffusion of point defects as centers of nonradiative recombination [6] would be essential unless certain circumstances.

It is known that anneal activation energy of room stable point defects in GaP is equal $E_{a 1}=1.5 \mathrm{eV}$ (for phosphorous vacancy $V_{p}$ ) and $E_{a 2}=2.0 \mathrm{eV}$ (for gallium vacancy $V_{\mathrm{Ga}}$ ) [6]. The number of possible defect transitions is very low at these temperatures and an atom is practically immobile. The probability of its capture by oscillating dislocation (according to [7])

$W\left(\gamma_{a}\right)=\frac{\gamma_{a}}{2 \pi\left[4+\gamma_{a}\right]} \cdot\left[8+\gamma_{a}^{2}\left(1-\exp \left(-\frac{2 \pi}{\gamma_{a}}\right)\right)\right]$

where $\gamma_{a}=\omega t, \omega-$ ultrasound frequency.

Calculated dependence of the probability of atom capture by dislocation as a function of defect activation energy $W\left(E_{a}\right)$ at $300 \mathrm{~K}$ is shown in Fig. 4. One can see that $W\left(E_{a}\right)$ increases with $E_{a}$ due to the increase of settled atom lifetime. It's also clear from this figure that it's incorrect to say about the defect diffusion coefficient increase at $E_{a 1}=1.5 \mathrm{eV}$ and $E_{a 2}=2.0 \mathrm{eV}$ due to its capture by dislocations at GaP as $W\left(\gamma_{a}\right)>1$. Dislocation capture model is unvalued in this case.

II. The dislocation exciton formation during ultrasound treatment. As it was mentioned earlier excitons are the main emission component of samples studied. A lot of studies had been published last years [7] which proved the existence of the electron and hole new excited state - the dislocation exciton. This is an asymmetric formation near dislocation axis with the lateral dimension $\varepsilon$ times less than the longitudinal one ( $\varepsilon-$ is the semiconductor permitivity). The spectrum of this state is caused by the existence of stopped bounds at dislocation edges to which acceptor levels at covalent crystals correspond.

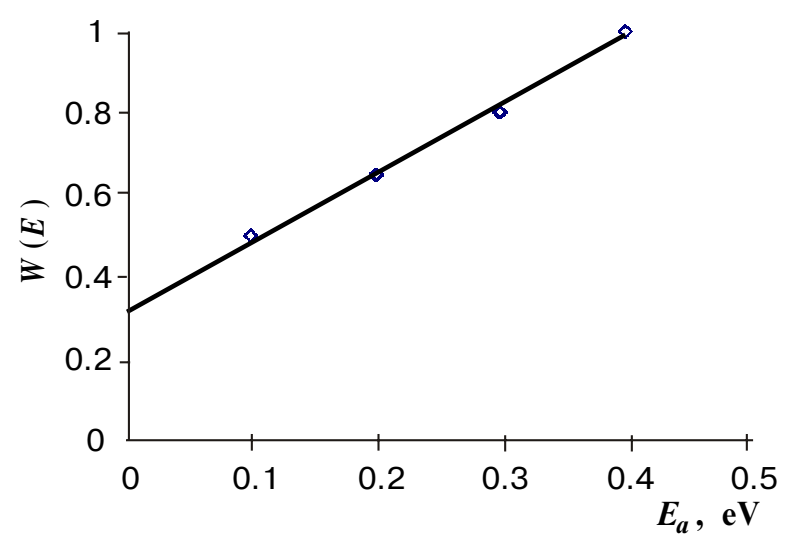

Fig. 4. Possibility of atom capture by dislocations as a function of the defect activation energy.

The wave function characteristic dimensions are equal [8]:

- at the longitudinal direction (parallel to dislocation axis):

$a_{\|}=\frac{\varepsilon h^{2}}{4 \pi^{2} \mu e^{2}}$,

- at the lateral direction (for an electron and a hole)

$a_{\perp}^{e, h}=\frac{h^{2}(1-\sigma)}{4 \pi m_{e, h} D_{e, h}(1-2 \sigma) b_{x}}$,

where reduced electron and hole mass is equal

$\mu=\frac{m_{e} \cdot m_{h}}{m_{e}+m_{h}}$

$D, \sigma$ and $b_{x}$-are deformation potential, Poisson coefficient, and Burgers vector, accordingly.

The ratio of $a_{\perp}$ to $a_{\|}$is equal:

$\frac{a_{\perp}}{a_{\|}}=\frac{(1-\sigma) \pi e^{2}}{(1-2 \sigma) b_{x} D \varepsilon} \approx \frac{1}{\varepsilon}<<1$,

Exciton total energy is

$E_{\text {ex.д. }}=-\frac{h^{2}}{8 \pi^{2} \mu a_{\|}^{2} n^{2}}-\varepsilon_{0}\left(\frac{h^{2}}{8 \pi^{2} m_{h}\left(a_{\perp}^{h}\right)^{2}}\right)$

Calculations made for GaP give next values:

$$
\begin{array}{ll}
a_{\|}=50 \AA ; & a_{\perp}{ }^{h}=1.4 \AA ; \\
a_{\perp}{ }^{e}=5.38 \AA ; & E_{\text {ex. }}=0.43 \mathrm{eV} .
\end{array}
$$

Let us estimate for the comparison the value of free exciton energy at GaP. According to [9],

$$
E_{e x}=-\frac{2 \pi^{2} M e^{4}}{h^{2} \varepsilon^{2}} \cdot \frac{1}{n^{2}}
$$

When $n=1$, calculation gives $E_{e x}=0.014 \mathrm{eV}$. The experimental value of $E_{e x}=0.021 \mathrm{eV}$. 
Using results of (7) and considering energy of forbidden band in $\mathrm{GaP}$ to be $E_{g}=2.24 \mathrm{eV}$ at room temperature, one can calculate the value of dislocation exciton energy:

$h v=E_{g}-E_{\text {ex. }}=2.24 \mathrm{eV}-0.43 \mathrm{eV}=1.81 \mathrm{eV}$.

The same value corresponds to the maximum of emission of excitons bounded on the $\mathrm{Zn}-\mathrm{O}$ complex. Thus, dislocation exciton emission at $\mathrm{GaP}$ is superposed on the main band $h v=1.8 \mathrm{eV}$ and become apparent as additional emission bands. Really, long-term ultrasound treatment promotes the appearing an additional amount of dislocations in its volume and their accumulation with mean density $\rho_{D} \sim 10^{7}-10^{8} \mathrm{~cm}^{-2}$ [9]. Up to the moment of dark line (DLD) and dark spot (DSD) defect formation these linear defects are mobile enough and can move along the crystal. As their geometrical size, electrical and deformation fields are unequal, so dislocation excitons with different wave function longitudinal and lateral parameters $a_{/ /}$and $a_{\perp}$ (and thus with different values of total energies) appear. It means that emission related with annihilation of such excitons can appear at different places of the spectrum investigated at energy interval of $h v=1.5-2.0 \mathrm{eV}$ and we can observe it as the result of emission of excitons, bounded on $\mathrm{Zn}-\mathrm{O}$ pair superposed on the main band (with spectrum maximum at $1.81 \mathrm{eV}$ ).

Time instability of these bands is evidently caused by ultrasonic induced dislocation interactions. It is known that dislocations can move not only under an acoustic stimulation but also without internal action, relaxing to the equilibrium state during certain time. Dislocation networks formation due to the dislocation flow superposition certainly promotes geometric size changes of separate dislocation excitons, which one can observe experimentally [10].

Spectral curve measured under acoustic dynamic mode gives an additional confirmation of the exciton origin of wandering lines in the spectrum of ultrasound treated diodes[11]. Almost complete thin structure vanishing in the $I(h v)$ curve and the quick restoring after an ultrasound stoppage are the evidence of the abrupt decrease of numbers of exciton annihilation events. Moving along the crystal the ultrasound wave (besides effects mentioned above) first of all destroys excitons bounded on dislocations. The structures of centers on which excitons are bounded does non vary.

\section{Conclusions}

Short ( $t<10 \mathrm{~min}$.) ultrasound treatment of $p$ - $n$ junctions of $\mathrm{GaP}$ light diodes causes small emission intensity increase (to $5 \%$ ) in the red band maximum $h v=1.81 \mathrm{eV}$. When the time of treatment increases, monotonous decrease of the intensity is observed. Gradual restoring of the diode emission ability occurs after ultrasonic action.

Positive effect of short time ultrasound treatment occurs due to the point defect absorption by dislocations moving along the crystal at ultrasound field. The improvement of emission characteristics of GaP light diode in this case is analogue to this which occurs in structures irradiated by ionizing radiation. As ultrasound is a simple and cheap treatment in comparison with radiation one can use it as convenient instrument for testing structure behavior under irradiation ( $\gamma$-rays, for example).

The phenomenon of the emission restoring of GaP diodes can't be explained as the defect capture by dislocations oscillated at ultrasound wave fields.

Spectrum of ultrasound degraded diodes possesses specific time changing wandering lines vanishing at the moment of ultrasound switch on (during acoustic - dynamic treatment). After ultrasound switch off these lines appear again. The appearing of these lines is caused by the dislocation exciton formation.

Ultrasound treatment decreases microplasma emission of reverse biased light diodes and promotes improvement of characteristics of optoelectronic devices.

\section{References}

1. R.V. Konakova, P. Kordos, Ju.A. Tchorik et. al., Reliability Prediction for the Semiconductor Avalanche Diodes (in Russian), Naukova dumka, Kyiv ( 1986).

2. I.B. Jermolovych, V.V. Milenin, R.V. Konakova et.al, Influence of ultrasound treatment on deformation effects and structure of local centres in a substrate and near contact regions of $\mathrm{M} / \mathrm{n}-\mathrm{n}^{+}-\mathrm{GaAs}(\mathrm{M}=\mathrm{Pt}, \mathrm{Cr}, \mathrm{W}$ ) structures (in Russian) // Fiz. Tech. Poluprov. 3( 4), pp.503-508 (1997).

3. A.S. Bakaj, N.P. Lazarev, Ultrasound influence on the diffusion of impurity incorporating atoms in solids (in Russian), // Phys. Tverd.Tela, 28(8), pp.2453-2457 (1986).

4. A.A. Dobrovolsky, V.V. Lemanov, A.B. Sherman, Interactions of acoustic surface waves in piezoelectrics with luminofors - new method of electroluminescence excitation (in Russian) // Pi's v Hz. Their. Fizz. 4(13), p.753 (1978).

5. A.Kelly, G. Gravs, Crystallography and defects in crystals (in Russian), Mir, Moscow, (1974).

6. O.F. Nemetz, V.V. Volkov, P.G. Litovchenko et. al., Radiation defects in GaP (in Russian) // Doklady AN SSSR, ser.A, 5, pp.47-50 (1988).

7. V.I. Sugakov, V.P. Khotjancev, Dislocation excitons in highly anizotropic crystals (in Russian) // Optika i spektroskopiya, 45(2), pp.301-305 (1978).

8. S.V. Bozokin, D.A. Parshyn, V.A. Kharchenko, Dislocation Mott's exciton (in Russian) // Phys. Tverd. Tela, 24(5), pp.14111415 (1982).

9. Z. Pankov, Optical processes in semiconductors (in Russian), Mir, Moscow (1973).

10. O.M. Gontaruk, V.F. Machulin, Y.M. Olikh et. al., About ultrasonic influence on relaxation-degradation fenomena in light emitting GaP p-n structures (in Russian) // Pis'ma v Zh. Tekhn. Fiz., 24(15), pp.64-68 (1998).

11. O.M. Gontaruk, M.B. Pinkovska, D.V., V.P.Tartachnyk et. al. (in Ukrainian) // Dopovidi NAN Ukrajiny, 11, pp.83-89 (1998). 